\title{
Motility evaluation of human spermatozoa
}

\author{
R. FINSY, J. PEETERMANS† and H. LEKKERKERKER \\ Vrije Universiteit Brussel, Faculteit van de Wetenschappen, \\ Dienst Theoretische Fysische Scheikunde, Brussels, Belgium
}

(Received 19 May 1979; revision received 13 August 1979)

\begin{abstract}
In this contribution we investigate the applicability of photon correlation techniques as a clinical tool for the evaluation of motility parameters of undiluted human sperm.

Measurements on semen samples, selected visually for 'good' motility, yielded reproducible estimates of the fraction motile spermatozoa and the mean speed of the motile cells.
\end{abstract}

\section{Introduction}

Fertility studies on human sperm have shown the need for objective measurements of motility parameters for the spermatozoa. Relevant parameters for the fertility of a spermatozoa population are the fraction $\alpha$ of motile cells in the sample and their mean speed $\bar{v}$. Photon correlation spectroscopy appears to be an accurate method for a rapid and objective determination of $\alpha$ and $\bar{v}$ [1]. A group of French investigators $[2,3,4]$ reported such motility measurements using a heterodyne mixing technique combined with a data analysis in the frequency domain. In this contribution we investigate the applicability of the homodyne light scattering technique with a data analysis in the time domain as a clinical tool for routine evaluations of the motility parameters $\alpha$ and $\bar{v}$ of undiluted human sperm.

We suppose that the scattered electric field is produced by only two different kinds of scatterers: motile and non-motile spermatozoa. Under this assumption the normalized autocorrelation function of the scattered field $g_{1}(\tau)$ can be written as

$$
\left|g_{1}(\tau)\right|=\alpha\left|g_{1}^{m}(\tau)\right|+(1-\alpha)\left|g_{1}^{d}(\tau)\right| .
$$

On the right hand side of this equation $g_{1}^{m}(\tau)$ and $g_{1}^{d}(\tau)$ are the field autocorrelation functions of the motile and the non-motile cells respectively. Jouannet et al. [4] have observed that the following isotropic speed distribution function

$$
P_{s}(v)=\frac{4 v}{\bar{v}^{2}} \exp \left[-\frac{2 v}{\bar{v}}\right]
$$

provides a good description for the motile spermatozoa. In equation (2) $P_{s}(v)$ is specified by the mean value $\bar{v}$ rather than by the modal value $\bar{v} / 2$ as done by Jouannet et al. Using equation (2) and assuming that the non-motile spermatozoa behave as identical brownian scatterers, $\left|g_{1}^{m}(\tau)\right|$ and $\left|g_{1}^{d}(\tau)\right|$ reduce to very simple expressions [5]. In particular $\left|g_{1}^{m}(\tau)\right|$ is given by

$$
\left|g_{1}^{m}(\tau)\right|=\left[1+\left(\frac{q \bar{v} \tau}{2}\right)^{2}\right]^{-1},
$$

where $q$ is the magnitude of the scattering vector.

† Scholarshipholder I.W.O.N.L. (Belgium). 
In a homodyne light scattering experiment, rather than the field autocorrelation function, one measures the intensity autocorrelation function. Since light scattered by a large number of scatterers such as spermatozoa in a semen sample obeys gaussian statistics [6], this function, i.e. in normalized form $g_{2}(\tau)$, can easily be expressed in terms of $\left|g_{1}(\tau)\right|$. In this way, the above model leads to a useful relation between $g_{2}(\tau)$ and the motility parameters $\alpha$ and $\bar{v}$ :

$$
g_{2}(\tau)=1+C\left|\alpha\left[1+\left(\frac{q \bar{v} \tau}{2}\right)^{2}\right]^{-1}+(1-\alpha) \exp \left[-\frac{|\tau|}{\tau_{d}}\right]\right|^{2}
$$

where $C$ is an instrumental factor and $\tau_{d}$ is the decay time of $g_{1}^{d}(\tau)$.

\section{Experimental}

The apparatus used for measuring $g_{2}(\tau)$ is essentially a Malvern 4300 Photon Correlation Spectrometer. Data analysis is performed on-line with a HewlettPackard HP 9825A calculator yielding estimates of the motility parameters of interest.

The scattering cells, containing the undiluted semen samples, are cylindrical glass vessels with inside diameter of about $3 \mathrm{~mm}$ and a volume of less than $0.1 \mathrm{ml}$.

At small scattering angles $\theta\left(\theta<20^{\circ}\right)$, the effect of unwanted mixing of the light scattered from the semen with specular light from the side walls of the sample holder was investigated. This was done by performing measurements on suspensions of polystyrene spheres (Dow Chemicals) at different scattering angles. From this it appeared that for angles down to $10^{\circ}$ the diameter of the latex spheres $(0 \cdot 234 \mu \mathrm{m})$ was confirmed by the measured diffusion coefficient assuming pure homodyne detection. Although measurements at $7^{\circ}$ apparently suffer from reflection by the walls of the scattering cells, the diameter of the spheres, calculated still assuming homodyne detection, was overestimated by no more than 20 per cent.

The uncertainty on the scattering angle due to off-centre alignment of the sample holder, was estimated not to exceed one degree in the worst case.

\section{Some results}

We first examined those semen samples which are characterized as 'good' according to visual observation by microscope (i.e. $\alpha \gtrsim 0 \cdot 6$ and an appreciation of a reasonably high value of $\bar{v}$ ). A typical result is given in figure 1 . The conclusion is that measurements on this category of sperm gives estimates of the fraction motile spermatozoa reproducible within 10 per cent and of the mean speed $\bar{v}$ of these cells reproducible within 5 per cent.

Determination of $\alpha$ and $\bar{v}$ as a function of the scattering angle in the range of $7^{\circ}$ to $20^{\circ}+$ revealed that

(a) $\alpha$ is independent within the experimental reproducibility of the scattering angle $\theta$; and

(b) $\bar{v}$ decreases systematically with increasing scattering angle $\theta$ even for very motile ( $\alpha$ almost 1$)$ semen samples.

Representative values are given in the caption of figure 2 showing the field autocorrelation function $\left|g_{1}(\tau)\right|$ plotted as a function of $q \tau$ for a very motile sample. Clearly the $q \tau$-scaling expected from equation (3) is not observed.

+ Scattering angles in the range $10^{\circ}$ to $20^{\circ}$ were used because at these angles most of the scattered intensity comes, as assumed in the model, from the spermatozoa themselves. 


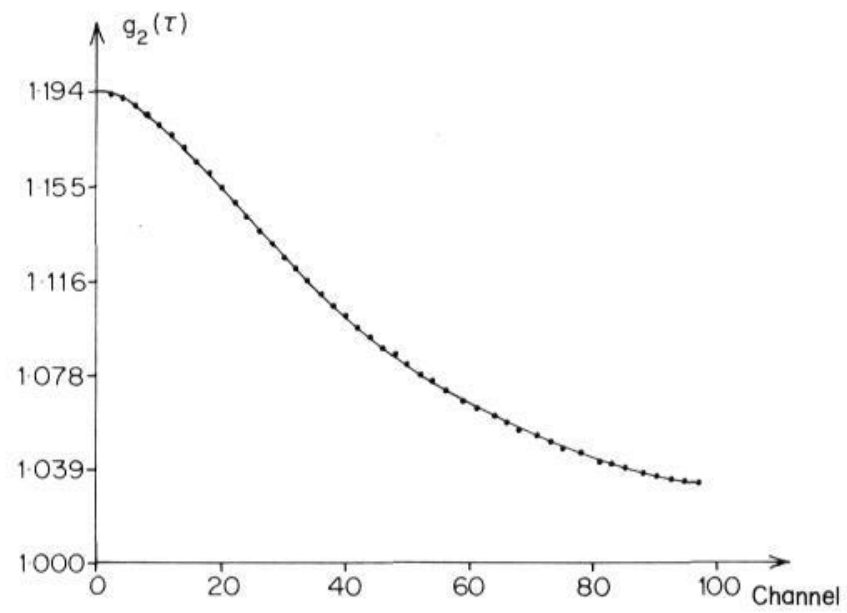

Figure 1. A typical normalized intensity autocorrelation function $g_{2}(\tau)$ for human spermatozoa. Dots are experimental points (one out of two) and the full line is a least squares fit with equation (4). Experimental conditions: scattering angle $\theta, 17^{\circ}$; delay time per channel, $100 \mu \mathrm{s}$; clip level, 1 ; temperature, $32^{\circ} \mathrm{C}$. Values obtained for the motility parameters: fraction motile spermatozoa $\alpha, 0 \cdot 54$; mean speed $\bar{v}, 81 \mu \mathrm{m} / \mathrm{s}$.

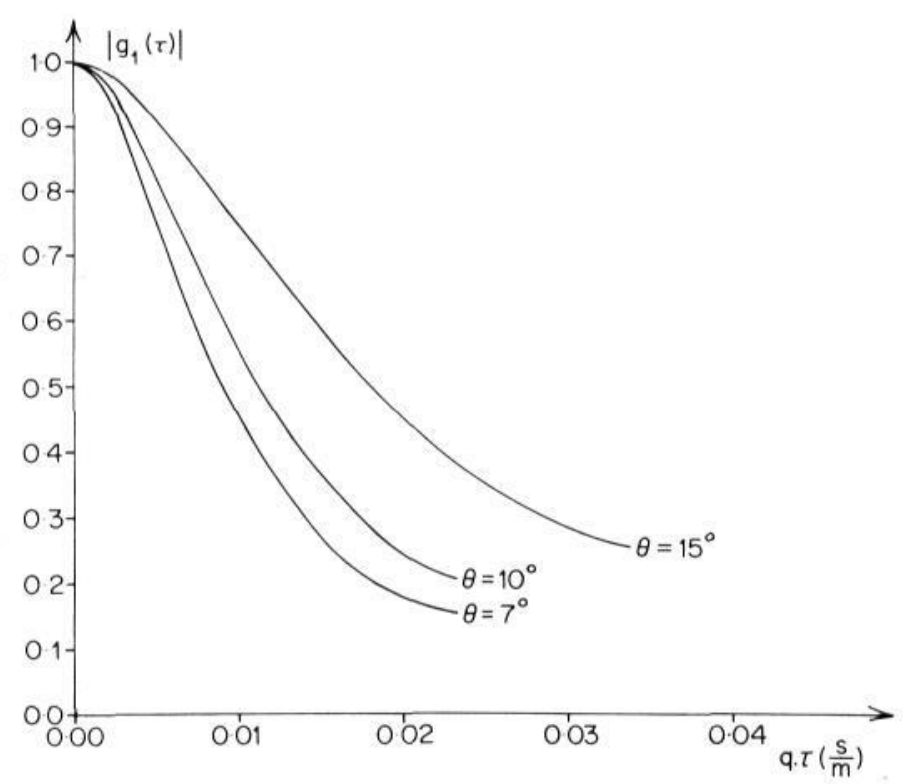

Figure 2. Field autocorrelation function $g_{1}^{m}(\tau)$ for a very motile sperm sample plotted as a function of $q \tau$. The three curves correspond to three different scattering angles: $\theta=7^{\circ}$, $\theta=10^{\circ}$, and $\theta=15^{\circ}$. Further experimental conditions were as follows. Temperature, $33^{\circ} \mathrm{C} ; \theta=7^{\circ}$ : clip level $=2$, delay time per channel $=150 \mu \mathrm{s}, \alpha=0.84, \bar{v}=263 \mu \mathrm{m} / \mathrm{s}$; $\theta=10^{\circ}:$ clip level $=1$, delay time per channel $=100 \mu \mathrm{s}, \alpha=0.79, \bar{v}=231 \mu \mathrm{m} / \mathrm{s} ; \theta=15^{\circ}$ : clip level $=0$, delay time per channel $=100 \mu \mathrm{s}, \alpha=0 \cdot 83, \bar{v}=154 \mu \mathrm{m} / \mathrm{s}$. 
In order to understand what is causing this behaviour, the following three assumptions were checked.

In the first place biasing due to specular light reflecting from the walls of the scattering cell was investigated. As reported in the experimental section, this effect is negligible for angles above $10^{\circ}$. Although a possible bias at smaller angles $\theta$ cannot be excluded, the point is that such a bias would lead to an apparent increase of $\bar{v}$ with $\theta$ instead of the observed decrease of $\bar{v}$ with increasing $\theta$.

Secondly, since the concentration of spermatozoa is rather high in an undiluted semen sample, multiple scattering may occur. In the case of significant multiple scattering, the observed intensity effectively corresponds to a spread of different scattering vectors instead of one fixed value determined by the scattering angle. The result would be that the intensity autocorrelation function $g_{2}(\tau)$ is less dependent on $\theta$ than equation (4) predicts. Whether or not multiple scattering is causing the observed non- $q \tau$-scaling, can be examined by diluting the sample. One hereby reduces the concentration of scattering objects and hence reduces the contribution of multiple scattering. Therefore measurements were performed on semen samples diluted 2, 4, 8 and 16 times with their own seminal liquid. Although this process does affect the motion of the spermatozoa $(\alpha$ and $\bar{v}$ are significantly altered with the dilution factor), the effect reported under $(b)$ above remained.

Thirdly, it should be stressed that the spermatozoa heads are large compared to the wavelength of the light, so that their shape and dimensions are to be taken into account. To do so we assumed them to be ellipsoidal and moving along their long semiaxis. The field autocorrelation function of light scattered by such particles can be written as follows under the Rayleigh-Gans criterion [7]

$$
g_{1}^{\mathrm{ell}}(\tau, q)=\frac{\int_{0}^{\infty} d v P_{s}(v) \int_{-1}^{+1} d \mu P(\mathbf{q}, \mu) \exp (i q v t \mu)}{\int_{-1}^{+1} d \mu P(\mathbf{q}, \mu)} .
$$

The speed distribution $P_{s}(v)$ is given by equation (2) and the orientation-dependent form factor $P(\mathbf{q}, \mu)$ is given by the expression

$$
P(\mathbf{q}, \mu)=\frac{g}{u^{4}}\left(\frac{\sin u}{u}-\cos u\right)^{2},
$$

where $u$ can be written as

$$
u=q \cdot\left[a^{2} \mu^{2}+b^{2}\left(1-\mu^{2}\right)\right]^{1 / 2} .
$$

Here $\mu$ is the cosine of the angle between the scattering vector $\mathbf{q}$ and the direction of the motion of the spermatozoa; $a$ and $b$ are respectively the large and the small semiaxis of the ellipsoidal spermatozoa heads. Notice that for a sphere (i.e. $a=b$ ) $g_{1}^{\text {ell }}(\tau, q)$ given by equation (5) reduces to $\left|g_{1}^{m}(\tau)\right|$ given by equation (3). In figure 3 , $g_{1}^{\text {ell }}(\tau, q)$ calculated with realistic values of $\bar{v}, a$ and $b$, is plotted as a function of $q \tau$ at three different angles $\theta$ (solid lines). The similarity with the experimental plots of figure 2 is striking.

To conclude this part of the discussion we can state that, although a small spurious effect may occur due to unwanted scattering from the sample holder walls at small scattering angles, the non-spherical shape of the spermatozoa-heads is mainly responsible for the experimentally observed increase of $\bar{v}$ with decreasing $\theta$. 
Comparing the field autocorrelation function $g_{1}^{m}(\tau)$ for spheres (the dotted line in figure 3 ) with that for ellipsoids, i.e. $g_{1}^{\text {ell }}(\tau, q)$ (the solid lines in figure 3 ), one can conclude that data analysis with equation (4) leads to systematic underestimations of $\bar{v}$. This effect due to the non-spherical shape of the spermatozoa, is the smallest at very small scattering angles. Measurements at these very small angles $\left(\theta<10^{\circ}\right)$ can however not be performed in the assumption of homodyne light scattering because of the mixing with light scattered by the sample holder. Furthermore data-analysis with $g_{1}^{\text {ell }}(\tau, q)$ instead of $g_{1}^{m}(\tau)$ (i.e. taking into account the shape and dimensions of the spermatozoa-heads) becomes rapidly non-feasible for routine-determinations of $\alpha$ and $\bar{v}$. A possible way to avoid this is to use some fixed representative values for $a$ and $b$ in equation (5), thus simplifying considerably the data analysis procedure with $g_{1}^{\text {ell }}(\tau, q)$.

Notwithstanding these complications, the technique yields relative values of relevant motility parameters of human spermatozoa that are meaningful in the sense that they allow one to compare different sperm samples regarding their motility.

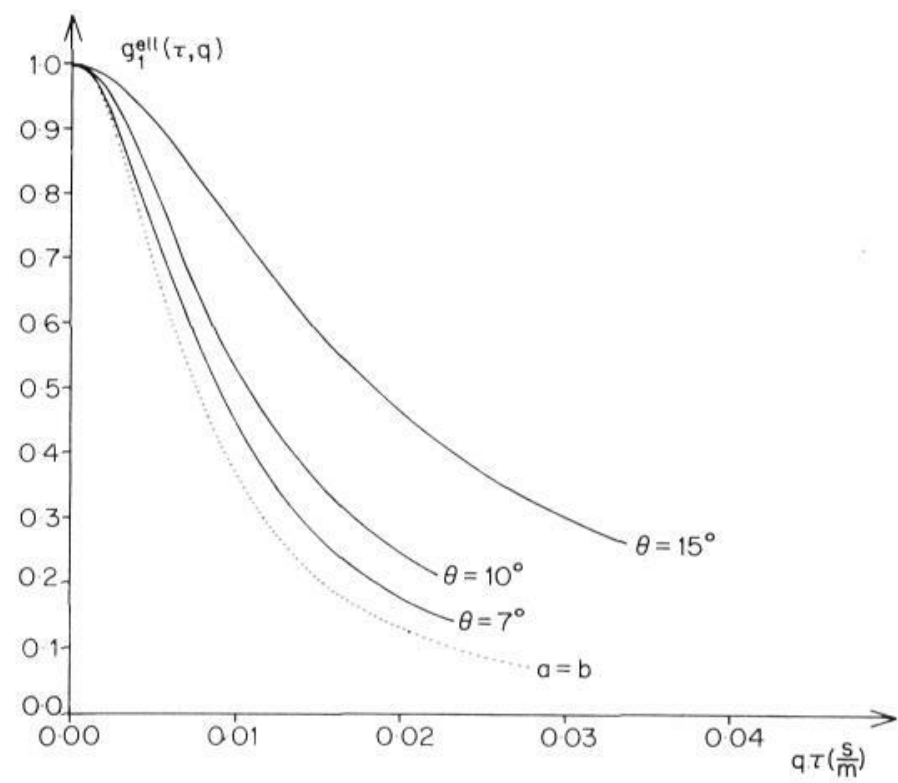

Figure 3. Solid Lines. Field autocorrelation function $g_{1}^{\text {ell }}(\tau, q)$ as calculated from equation (5) plotted as a function of $q \tau$. The three curves correspond to three different scattering angles: $\theta=7^{\circ}, \theta=10^{\circ}$ and $\theta=15^{\circ}$. Delay times: $\theta=7^{\circ}$, delay time per channel $=150 \mu \mathrm{s}$; $\theta=10^{\circ}$, delay time per channel $=100 \mu \mathrm{s} ; \theta=15^{\circ}$, delay time per channel $=100 \mu \mathrm{s}$. Chosen values: mean speed, $\bar{v}=260 \mu \mathrm{m} / \mathrm{s}$; large semi-axis, $a=1.50 \mu \mathrm{m}$; small semi-axis, $b=1.00 \mu \mathrm{m}$. Dotted Line. Field autocorrelation function related to spheres, i.e. $\left|g_{1}^{m}(\tau)\right|$ as calculated from equation (3) plotted as a function of $q \tau$ and for a mean speed $\bar{v}=260 \mu \mathrm{m} / \mathrm{s}$.

\section{Acknowledgment}

One of us (Joy Peetermans) would like to thank the Instituut tot Aanmoediging van het Wetenschappelijk Onderzoek in Nijverheid en Landbouw (I.W.O.N.L.) for financial support. 
Dans cette contribution nous étudions l'application de la technique de correlation de photons comme instrument clinique pour la détermination de paramètres de mobilité des spermatozoïdes humaines dans le sperme non dilué.

Des mesures sur des spermes, selectés par examen visuel pour une 'bonne' mobilité, donnent des estimations reproducibles de la fraction de spermatozoïdes mobiles et de leur vitesse moyenne.

Wir untersuchen in diesem Beitrag die Anwendbarkeit von Photon-Korrelationstechniken als klinisches Werkzeug zur Bestimmung von Motilitätsparametern unverdunnter menschlicher Spermatozoen.

Messungen an visuell nach 'guter' Motilität ausgewählten Samenproben ergaben reproduzierbare Abschätzungen der teilmotilen Spermatozoen und die mittlere Geschwindigkeit der motilen Zellen.

\section{References}

[1] Cummins, H. Z., 1977, Photon Correlation Spectroscopy and Velocimetry, edited by H. Z. Cummins and E. R. Pike (New York: Plenum Press), p. 200.

[2] Berge, P., and Dubois, M., 1973, Rerue Phys. appl., 8, 89.

[3] Dubois, M., Jouannet, P., Berge, P., Volochine, B., Serres, C., and David, G., 1975, Annls Phys. Biol. Méd., 9, 19.

[4] Jounnnet, P., Volochine, B., Deguent, P., Serres, C., and David, G., 1977, Andrologia, 9,36 .

[5] Finsy, R., Peetermans, J., and Lekkerkerker, H., 1979, Biophys. J., 27, 187.

[6] Shimizu, H., and Matsumato, G., 1976, Optics Commun., 16, 197.

[7] Chen, S. H., Holz, M., and Tartaglia, P., 1977, Appl. Optics, 16, 187. 